\title{
Influência da Temperatura, da Massa Molar e da Distribuição de Massa Molar na Tensão Superficial de PS, PP e PE: Experimento e Teoria
}

\author{
José C. Moreira, Nicole R. Demarquette, Renato N. Shimizu \\ Departamento de Engenharia Metalúrgica e de Materiais, EPUSP
}

\author{
Mazen K. Samara, Musa R. Kamal \\ Departamento de Engenharia Química, McGill University
}

Resumo: Neste trabalho a influência da temperatura, da massa molar $\left(\bar{M}_{n}\right)$ e da distribuição de massa molar (MWD) na tensão superficial de poliestireno (PS) foi avaliada utilizando o método da gota pendente. A influência da temperatura na tensão superficial de polipropileno isotático (i-PP) e de polietileno de baixa densidade (PELBD) também foi estudada aqui. As teorias de Patterson-Rastogi e Dee-Sauer foram utilizadas em conjunção com a teoria de equação de estado de Flory, Orwoll, e Vrij (FOV) para prever a tensão superficial $(\gamma)$ utilizando dados de pressão-volume-temperatura (PVT) dos polímeros. Ambas teorias prevêem que a tensão superficial diminui linearmente com o aumento da temperatura e aumenta com a massa molar concordando com os resultados experimentais. Entretanto, ambas teorias subestimam a mudança de entropia de formação de superfície por unidade de área a volume constante para sistemas poliméricos de baixa massa molar e polidisperso e subestimam o efeito da distribuição de massa molar na tensão superficial

Palavras-chaves: Tensão superficial, poliestireno, polietileno, polipropileno.

\section{Influence of Temperature, Molecular Weight, and Molecular Weight Dispersity on the Surface Tension of PS, PP, and PE: Experiments and Theoretical Analysis}

Abstract: In this work, the influence of temperature, molecular weight $\left(\bar{M}_{n}\right)$ and molecular weight dispersity (MWD) on the surface tension of polystyrene (PS) was evaluated using the pendant drop method. The influence of temperature on the surface tension of isotatic polypropylene (i-PP) and of linear low density polyethylene (LLDPE) was also studied here. Patterson-Rastogi and Dee and Sauer theories were used in conjunction with the Flory, Orwoll, and Vrij (FOV) equation of state to predict surface tension $(\gamma)$ using bulk pressure-volumetemperature (PVT) data. Both theories predicted that surface tension decreases linearly with increasing temperatures and increases with molecular weight corroborating the experimental data. However, both theories underestimated the entropy change of surface formation per unit area at constant volume for low molecular weight and polydisperse systems and underestimated the effect of molecular weight dispersity on surface tension.

Keywords: Surface tension, polystyrene, polyethylene, polypropylene.

\section{Introdução}

O conhecimento da tensão superficial de polímeros no estado fundido é muito importante na tecnologia de plásticos, tintas, têxteis e adesivos. A tensão superficial está diretamente correlacionada a conceitos de molhabilidade e adesão ${ }^{[1-4]}$. Infelizmente, existem poucos dados de tensão superficial de

Autor para correspondência: Nicole R. Demarquette, Departamento de Engenharia Metalúrgica e de Materiais, EPUSP, Av. Prof. Mello Morais, 2463, CEP: 05508-900, São Paulo, SP. E-mail: nick@usp.br 
polímeros no estado fundido devido às dificuldades experimentais encontradas para determinar este parâmetro ${ }^{[5]}$.

Recentemente, várias teorias termodinâmicas foram desenvolvidas para avaliar a tensão superficial de polímeros. Elas basicamente podem ser divididas em dois grupos: (i) as baseadas na teoria de gradiente quadrático primeiramente desenvolvida por CahnHilliard $(\mathrm{CH})$ que correlaciona a energia de superfície com a densidade e o gradiente quadrático de perfil de densidade numa superfície ${ }^{[1,2]}$ e (ii) as teorias de modelo de célula que se fundamentam no fato que a tensão superficial segue um princípio de estados correspondentes ${ }^{[1,2]}$. Por causa das dificuldades encontradas na determinação experimental da tensão superficial de polímeros no estado fundido é de grande interesse testar a validade destas teorias.

Neste trabalho a influência da temperatura, da massa molar e da distribuição de massa molar na tensão superficial de PS foi estudada. A influência da temperatura na tensão superficial de polipropileno isotático (i-PP) e de polietileno linear de baixa densidade (PELBD) também foi estudada aqui. Os resultados experimentais foram comparados com as predições teóricas de Patterson e Rastogi ${ }^{[7]}$ e Dee e Sauer ${ }^{[1,2]}$.

\section{Considerações teóricas}

Roe $^{[6]}$ e Patterson e Rastogi ${ }^{[7]}$ mostraram a existência de princípio de estados correspondentes para a tensão superficial de líquidos de moléculas pequenas e de vários polímeros. Eles observaram a existência de uma curva universal quando plotaram a tensão superficial reduzida em função da temperatura reduzida para todos estes materiais. A tensão superficial reduzida foi definida por Patterson e Rastogi como ${ }^{[7]}$ :

$$
\tilde{\gamma}=\frac{\gamma}{\gamma^{*}}=\frac{\gamma}{(k / \alpha)^{1 / 3} \beta^{2 / 3}}
$$

onde $\tilde{\gamma}$ e $\gamma^{*}$ são a tensão superficial reduzida e de redução, $\gamma$ é a medida de tensão superficial, k é a constante de Boltzmann, $\alpha$ é a expansividade térmica e $\beta$ é a compressibilidade térmica. A temperatura reduzida foi definida como $\alpha \mathrm{T}$.

Em seus estudos, Patterson e Rastogi ${ }^{[7]}$ usaram valores de tensão superficial e dados de PVT de diferentes fontes da literatura e observaram uma variação de $7 \%$ sobre a curva universal.
Para estudar o possível desvio de uma curva universal dentro de um grupo de moléculas com similar estrutura química mas com diferença em massa molar e arquitetura, Dee e Sauer ${ }^{[1,2]}$ utilizaram dados de PVT e tensão superficial mais precisos (agora disponíveis por causa do desenvolvimento de novos métodos experimentais) e geraram uma curva universal dentro de cada série de polímeros plotando $\tilde{\gamma}=\frac{\gamma}{\gamma^{*}}=\frac{\gamma}{P^{* 2 / 3} T^{* 1 / 3}(c k)^{1 / 3}}$ (onde c é um parâmetro de rede que representa o número de graus de liberdade do mero na rede) como uma função de $\widetilde{T}=\frac{T}{T^{*}}$.

A existência de um princípio de estados correspondentes permitiu a Patterson e Rastogi ${ }^{[7]}$ e Dee e Sauer ${ }^{[1,2]}$ usarem um formalismo de célula para gerar uma equação que previa a tensão superficial reduzida de um polímero em função das propriedades termodinâmicas daquele polímero. Em ambas teorias, os autores consideraram uma célula de rede para gerar uma função de partição que é utilizada para calcular a energia livre do sistema não homogêneo com uma superfície livre que está diretamente relacionada com a tensão superficial. Uma expressão da tensão superficial em função de parâmetros microscópicos é então obtida. A rede considerada foi descrita em detalhes em várias referências ${ }^{[1,2]}$ e é baseada no formalismo desenvolvido por Lennard-Jones e Devonshire para descrever as propriedades de líquidos de moléculas pequenas $^{[8]}$.

A Figura 1 mostra um corte bidimensional da rede considerada em ambos os modelos. Cada bola preta representa um segmento de polímero ou mero que pode se mover dentro da célula mas não pode se mover entre as células. A conectividade do polímero é considerada assumindo-se que cada mero tem $3 \mathrm{c}$ graus de liberdade. Cada segmento de polímero tem um volume

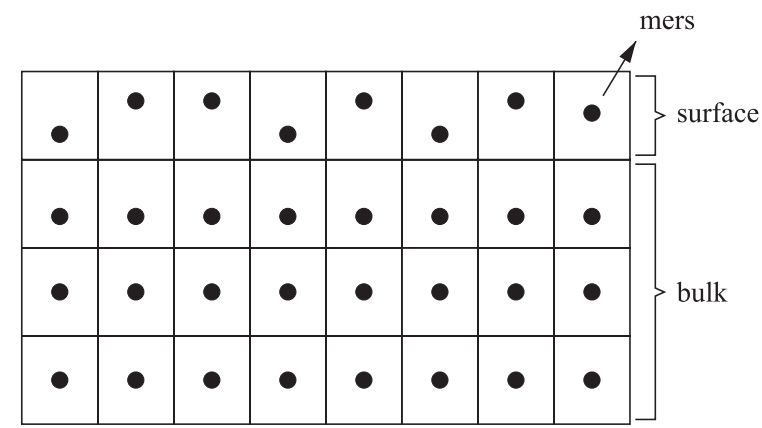

Figura 1. Diagrama esquemático de um modelo de rede de um estado condensado com uma superfície livre no topo. 
de núcleo duro $v^{*}$. O volume não ocupado pelos segmentos de polímero corresponde ao volume livre. Pode ser visto da Figura 1 que a rede pode ser dividida em duas zonas: o "bulk" e a superfície e que o volume livre associado a cada segmento é diferente dependendo se o mero está localizado na superfície ou no bulk. Assim sendo, para relacionar os parâmetros microscópicos do modelo com as propriedades do sistema real, a equação de estado de FloryOrwoll-Vrij (FOV) ${ }^{[9]}$ foi utilizada e será descrita mais adiante no artigo.

Patterson e Rastogi ${ }^{[7]}$ estenderam a teoria de Prigogine-Saraga ${ }^{[10]}$ para prever a tensão superficial de polímeros líquidos e geraram uma expressão para a tensão superficial reduzida dada por:

$$
\begin{aligned}
& \tilde{\gamma}=\frac{m}{\widetilde{V}^{5 / 3}}-\frac{\left(1-\widetilde{V}^{-1 / 3}\right)}{\widetilde{V}^{5 / 3}} \ln \left[\frac{\left(\widetilde{V}^{1 / 3}-0.5 b_{P R}\right)}{\left(\widetilde{V}^{1 / 3}-b_{P R}\right)}\right] \\
& \tilde{\gamma}=\frac{\gamma}{\gamma^{*}}=\frac{\gamma}{P^{* 2 / 3} T^{* 1 / 3}(k c)^{1 / 3}}
\end{aligned}
$$

onde $\widetilde{V}, \mathrm{P}^{*}$ e $\mathrm{T}^{*}$ são volume reduzido, pressão e temperatura de redução que podem ser inferidos da equação de estado de FOV que será mostrada abaixo; $\mathrm{m}$ representa a fração de vizinhos perdidos por um segmento de cadeia quando se move do bulk para a superfície, c foi considerado igual a 1 e $b_{\mathrm{PR}}$ é o fator de empacotamento inerente a teoria ${ }^{[7]}$. Dee e Sauer ${ }^{[1,2]}$ utilizaram uma definição diferente do volume livre dos meros na camada superficial (equação 7a da referência 1) e obtiveram a seguinte expressão para a tensão superficial reduzida de um polímero:

$$
\begin{aligned}
& \tilde{\gamma}=\frac{\gamma}{\gamma^{*}}=\frac{m}{\widetilde{V}^{5 / 3}}-\frac{\widetilde{T}}{\widetilde{V}^{2 / 3}} \ln \left[\frac{\left(b_{D S} \widetilde{V}^{1 / 3}\right)}{\left(\widetilde{V}^{1 / 3}-1\right)}\right] \\
& \tilde{\gamma}=\frac{\gamma}{\gamma^{*}}=\frac{\gamma}{P^{* 2 / 3} T^{* 1 / 3}(k c)^{1 / 3}}
\end{aligned}
$$

onde $b_{\mathrm{DS}}$ é um parâmetro que correlaciona o volume livre dos meros da camada superficial com os dos meros do "bulk". $b_{\mathrm{DS}}$ pode ser relacionado com a espessura da camada interfacial, $\varphi$, de acordo com:

$$
b_{D S}=\frac{\varphi}{\left(v^{*} \widetilde{V}\right)^{1 / 3}}
$$

onde $v^{*}$ é volume de núcleo duro do mero.
Para ambas teorias o primeiro e o segundo termo no lado direito da equação (equações $2 \mathrm{a}$ e $3 \mathrm{a}$ ) representam as contribuições entálpicas e entrópicas para a tensão superficial, respectivamente.

Patterson e Rastogi[ ${ }^{[7]}$ recomendaram o uso de $\mathrm{m}=0,25$ e $\mathrm{b}=1$ porque outros valores levariam a valores baixos de energia de superfície e entropia. De acordo com Dee e Sauer ${ }^{[1,2]}$ os valores de b e m que produzem o melhor ajuste aos resultados experimentais são, respectivamente, da ordem de 2 e 0,52 .

Para inferir a tensão superficial utilizando as equações (2) Patterson e Rastogi - e (3) - Dee e Sauer é necessário conhecer os parâmetros reduzidos e de redução. Em ambas teorias, eles podem ser obtidos por diferenciação da equação de estado de FloryOrwoll-Vrij (FOV) ${ }^{[9]}$ :

$$
\frac{\widetilde{P} \widetilde{V}}{\widetilde{T}}=\frac{\widetilde{V}^{1 / 3}}{\widetilde{V}^{1 / 3}-1}-\frac{1}{\widetilde{V} \widetilde{T}}
$$

onde $\widetilde{P}, \widetilde{V}$ e $\widetilde{T}$ são parâmetros reduzidos de pressão, volume e temperatura, respectivamente.

A diferenciação desta equação de estado de FOV com respeito a temperatura, a pressão constante e volume zero e com respeito a temperatura, a volume constante e pressão zero geram o volume e a pressão reduzida, dadas pelas equações abaixo:

$$
\begin{aligned}
& \widetilde{V}=\frac{v_{s p}}{v_{s p}^{*}}=\left[\frac{1+T \alpha}{1+4 T \alpha / 3}\right]^{-3} \\
& \widetilde{P}=P / P^{*}=P /\left(\widetilde{V}^{2} T \alpha / \beta\right)=P /\left(\widetilde{V}^{2} T \lambda\right)
\end{aligned}
$$

onde $\alpha=\partial \ln V /\left.\partial T\right|_{P}$ é o coeficiente de expansão térmica; $\lambda=\partial p /\left.\partial T\right|_{v} e ́$ o coeficiente de pressão térmica e $\beta=-\partial \ln V /\left.\partial p\right|_{T}$ é a compressibilidade isotérmica.

Então, a temperatura reduzida pode ser obtida algebricamente utilizando as equações (5) e (6):

$$
\widetilde{T}=T / T^{*}=T \widetilde{V}^{1 / 3} /\left(\widetilde{V}^{1 / 3}-1\right)
$$

Combinando as equações (6a-6c) com valores experimentais conhecidos de $v_{s p}(T), \alpha(T)$, e $\lambda(T)$ ou $\beta(T)$, é suficiente para determinar os parâmetros de redução e reduzidos $\mathrm{v}_{s p}^{*}, P^{*}, T^{*} \mathrm{e} \widetilde{P}, \widetilde{T}, \widetilde{V}$, respectivamente.

Neste trabalho, a validade das teorias foi verificada para poliestireno de massa molar e de distribuição de massa molar variada e para várias 
poliolefinas. Para inferir os parâmetros reduzidos e de redução de poliestireno polidisperso, uma lei de mistura simples foi usada, seguindo Dee ${ }^{[11]}$ e foi assumido que:

$$
\begin{aligned}
\widetilde{V} & =\sum x_{i} \widetilde{V}_{i} \\
\widetilde{T} & =\sum x_{i} \widetilde{T}_{i} \\
T^{*} & =\sum x_{i} T_{i}^{*} \\
P^{*} & =\sum x_{i} P_{i}^{*}
\end{aligned}
$$

onde, $P^{*}, T^{*}$ e $\widetilde{T}, \widetilde{V}$ são os parâmetros de redução e reduzidos do sistema multicomponente; $\widetilde{V}_{\mathrm{i}}, \widetilde{T}_{\mathrm{i}}$ e $T_{i}^{*}$, $P_{i}^{*}$ são parâmetros reduzidos e de redução das espécies $\mathrm{i} ; \mathrm{x}_{\mathrm{i}}$ é a fração molar das espécies $\mathrm{i}$.

\section{Experimental}

\section{Materiais}

As influências da temperatura, da massa molar e da distribuição de massa molar foram estudadas experimentalmente para poliestireno, polipropileno e polietileno.

a) PS: Amostras monodispersas com massa molar variando de $3.000 \mathrm{~g} / \mathrm{mol} \mathrm{a} 700.000 \mathrm{~g} / \mathrm{mol}$.

b) PP: Dois tipos de polipropileno isotático foram usados: i-PP ${ }_{1}: \bar{M}_{n}=70.400 \mathrm{~g} / \mathrm{mol} \mathrm{e} \mathrm{I}=4,17$ e i-PP ${ }_{2}$ : $\bar{M}_{n}=80.100 \mathrm{~g} / \mathrm{mol} \mathrm{e} \mathrm{I}=4,32 \mathrm{um}$ (I representa o índice de polidispersidade). A diferença entre as duas amostra estava na presença de aditivos ${ }^{[13]}$.
Tabela 1. Poliestireno Monodisperso e Poliolefinas utilizadas neste trabalho

\begin{tabular}{cccc}
\hline Polímero & $\bar{M}_{n}$ & $I=\bar{M}_{w} / \bar{M}_{n}$ & Fornecedor \\
\hline$P S_{\text {mono }}^{2.180}$ & 2.180 & 1,03 & Aldrich \\
$P S_{\text {mono }}^{3.400}$ & 3.400 & 1,09 & Aldrich \\
$P S_{\text {mono }}^{12.400}$ & 12.400 & 1,06 & Aldrich \\
$P S_{\text {mono }}^{18.100}$ & 18.100 & 1,07 & Aldrich \\
$P S_{\text {mono }}^{29.100}$ & 29.100 & 1,08 & Aldrich \\
$P S_{\text {mono }}^{41.200}$ & 41.200 & 1,07 & Aldrich \\
$P S_{\text {mono }}^{107.200}$ & 107.200 & 1,07 & Aldrich \\
$P S_{\text {mono }}^{200.600}$ & 200.600 & 1,11 & Aldrich \\
$P S_{\text {mono }}^{339.500}$ & 339.500 & 1,16 & Aldrich \\
$P S_{\text {mono }}^{678.000}$ & 678.000 & 1,12 & Aldrich \\
i-PP 1 & 70.400 & 4,17 & OPP Química \\
i-PP & 80.100 & 4,32 & OPP Química \\
LLDPE $^{2}$ & 91.800 & 4,42 & OPP Química \\
\hline
\end{tabular}

c) PELBD: Um polietileno linear de baixa densidade com $\bar{M}_{n}=91.800 \mathrm{~g} / \mathrm{mol} \mathrm{e} \mathrm{I}=4,42$ (I representa o índice de polidispersidade).

Para estudar a influência da distribuição de massa molar de poliestireno (PS) na tensão superficial, amostras de poliestireno foram misturadas em diferentes proporções de peso para obter amostras de poliestireno com polidispersidade variada mas mantendo-se a massa molar numérica média constante $(\approx 18.100 \mathrm{e} \approx 107.200 \mathrm{~g} / \mathrm{mol})$.

As Tabelas 1 a 3 fornecem os detalhes a respeito dos materiais e suas características moleculares. Os deta-

\begin{tabular}{|c|c|c|c|c|c|c|c|c|}
\hline \multirow[b]{2}{*}{ Blenda } & \multirow[b]{2}{*}{ Polidispersidade } & & \multicolumn{6}{|c|}{ Porcentagem (\%) em peso dos diferentes PS usados na blenda } \\
\hline & & $\mathrm{PS}^{2180}$ & $\mathrm{PS}^{3700}$ & $\mathrm{PS}^{12400}$ & $\mathrm{PS}^{18100}$ & $\mathrm{PS}^{29100}$ & $\mathrm{PS}^{41200}$ & $\mathrm{PS}^{107200}$ \\
\hline$P S \begin{array}{l}18100 \\
\text { poli } 3\end{array}$ & 1.14 & & & 33.8 & 25 & 41.2 & & \\
\hline$P S \quad \begin{array}{l}18100 \\
\text { poli } 5\end{array}$ & 1.55 & & 5.2 & 18.6 & 13.8 & 22.6 & 39.8 & \\
\hline$P S \underset{p o l i 7}{18100}$ & 2.68 & 3.1 & 3.6 & 13.1 & 9.6 & 15.8 & 27.9 & 26.9 \\
\hline
\end{tabular}
lhes da caracterização dos polímeros e obtenção das blendas podem ser encontrados nas referências 12 e14.

\begin{tabular}{|c|c|c|c|c|c|c|c|c|}
\hline \multirow[b]{3}{*}{ Blenda } & \multirow[b]{3}{*}{ Polidispersidade } & \multirow{2}{*}{\multicolumn{7}{|c|}{ Porcentagem $(\%)$ em peso dos diferentes PS usados na blenda }} \\
\hline & & & & & & & & \\
\hline & & $\mathrm{PS}^{18100}$ & $\mathrm{PS}^{29100}$ & $\mathrm{PS}^{41200}$ & $\mathrm{PS}^{107200}$ & $\mathrm{PS}^{200600}$ & $\mathrm{PS}^{339500}$ & $\mathrm{PS}^{679000}$ \\
\hline PS $\begin{array}{l}107200 \\
\text { poli } 3\end{array}$ & 1.40 & & & 16.9 & 25 & 58.1 & & \\
\hline PS $\begin{array}{l}1007200 \\
\text { poli } 5\end{array}$ & 1.93 & & 9.1 & 9.3 & 13.7 & 32 & 35.9 & \\
\hline PS $\begin{array}{l}107200 \\
\text { poli } 7\end{array}$ & 2.98 & 4.4 & 6.4 & 6.4 & 9.6 & 22.4 & 25.1 & 25.6 \\
\hline
\end{tabular}

Tabela 2. Composição de Poliestireno Polidisperso para $=18.100 \mathrm{~g} / \mathrm{mol}$

Tabela 3. Composição de Poliestireno Polidisperso para $=107.200 \mathrm{~g} / \mathrm{mol}$ 


\section{Tensão Superficial}

Os resultados de tensão superficial foram obtidos com um aparelho da gota pendente descrito em um outro trabalho ${ }^{[15]}$. Ele consiste essencialmente de uma câmara experimental aquecida onde a gota foi formada, de um sistema óptico para capturar a imagem da gota pendente e de um sistema de aquisição de dados num microcomputador pentium PC para inferir a tensão superficial do perfil da gota. Um procedimento de digitalização automático foi usado nas medidas de tensão superficial apresentadas neste trabalho. A imagem da gota pendente foi digitalizada por um frame grabber interligado num microcomputador PC e as medidas de tensão superficial foram analisadas on-line. A análise dos experimentos conduzidos aqui foi feita utilizando algoritmos desenvolvidos por Anastasiadis et al. ${ }^{[16]} \mathrm{e}$ Demarquette $\mathrm{e}$ $\mathrm{Kamal}^{[17]}$. Estes algoritmos e procedimentos experimentais foram descritos conforme referência 15.

\section{Dados de PVT}

Os dados de pressão, volume e temperatura (PVT) para as amostras de poliestireno (PS) foram obtidas da literatura ${ }^{[18]}$ e para as poliolefinas foram obtidos utilizando um dilatômetro PVT da Gnomix (Denver Co). Os ensaios foram realizados seguindo os procedimentos de Capt e Kamal ${ }^{[19]}$.

\section{Resultados e Discussão}

\section{Dados de PVT}

A Figura 2 mostra um gráfico típico de PVT. Neste caso são mostrados os dados de PVT de polipropileno isotático (i-PP 1 ). A incerteza dos resultados é de $0,003 \mathrm{~cm}^{3} / \mathrm{g}$.

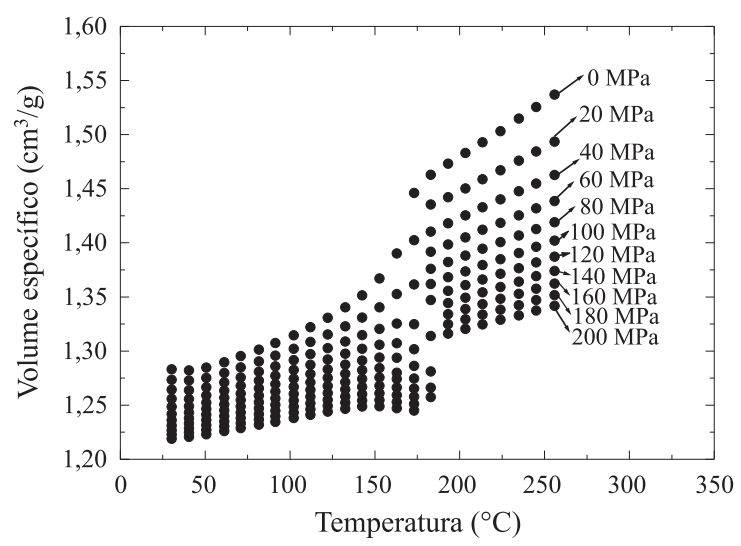

Figura 2. Volume específico vs temperatura de $\mathrm{i}-\mathrm{PP}_{1}$
Neste trabalho, o parâmetro reduzido $\widetilde{V}$ para o volume e os parâmetros de redução $P^{*}$ e $T^{*}$ para a pressão e temperatura, respectivamente, das amostras de poliestireno, polipropileno e polietileno foram inferidos das equações (6a) a (6c).

No caso de sistemas polidispersos os parâmetros reduzidos e de redução para a pressão, volume e temperatura foram avaliados utilizando a regra de mistura das equações (7a) a (7d). O tratamento dos dados de PVT e a obtenção de parâmetros reduzidos e de redução é mostrado em detalhes em um outro trabalho ${ }^{[20]}$.

\section{Influência da Temperatura}

A Figura 3 mostra uma comparação dos dados experimentais e das predições teóricas para a influência da temperatura na tensão superficial de polipropileno. Os diferentes símbolos representam os dados experimentais e as linhas as predições teóricas. Pode ser visto da Figura 3 que ambas teorias, Patterson e Rastogi e Dee e Sauer, prevêem que a tensão superficial de polipropileno diminui linearmente com o aumento da temperatura. Isto concorda com os dados experimentais. Contudo, ambas teorias não prevêem em valores absolutos os valores de tensão superficial. A teoria de Dee e Sauer parece superestimar a tensão superficial enquanto que a teoria de Patterson e Rastogi parece subestimá-la. Isto foi visto para todos os polímeros estudados aqui.

A Tabela 4 apresenta os coeficientes obtidos por ajuste linear dos dados experimentais e das predições teóricas de tensão superficial em função da temperatura para poliestireno, polipropileno e polietileno; a representa uma extrapolação da tensão superficial em $0{ }^{\circ} \mathrm{C}$ e b representa $-\frac{\partial \gamma}{\partial T}$. Pode ser visto da Tabela 4 que $-\frac{\partial \gamma}{\partial T}$ é constante em função da massa molar e em

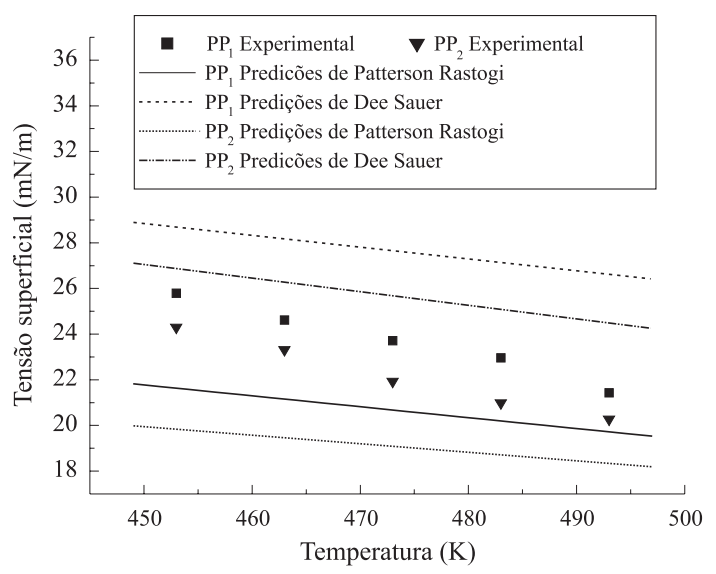

Figura 3. Tensão superficial vs temperatura para i-PP ${ }_{1}$ e i-PP 
Tabela 4. Coeficientes de regressão linear da dependência da tensão superficial em função da temperatura dos dados experimentais e das predições teóricas de Patterson-Rastogi e Dee-Sauer: $\gamma=\mathrm{a}-\mathrm{bt}$

\begin{tabular}{|c|c|c|c|c|c|c|c|c|c|}
\hline \multirow[b]{2}{*}{ Polímero } & \multicolumn{3}{|c|}{ Experimental } & \multicolumn{3}{|c|}{ Theory of Patterson-Rastogi } & \multicolumn{3}{|c|}{ Theory of Dee-Sauer } \\
\hline & a (dyne/cm) & $\mathrm{b}\left(\right.$ dyne $\left./ \mathrm{cm}^{\circ} \mathrm{C}\right)$ & $r^{2}$ & $\mathrm{a}($ dyne $/ \mathrm{cm})$ & $\mathrm{b}\left(\right.$ dyne $\left./ \mathrm{cm}^{\circ} \mathrm{C}\right)$ & $\mathrm{r}^{2}$ & $\mathrm{a}($ dyne $/ \mathrm{cm})$ & $\mathrm{b}\left(\right.$ dyne $\left./ \mathrm{cm}^{\circ} \mathrm{C}\right)$ & $r^{2}$ \\
\hline$P S_{\text {mono }}^{3.400}$ & 49,17 & 0,0956 & 0,9940 & 56,3 & 0,0643 & 1,000 & 73,0 & 0,0845 & 0,998 \\
\hline$P S_{\text {mono }}^{12.400}$ & 45,42 & 0,0763 & 0,9851 & 56,0 & 0,0623 & 1,000 & 68,1 & 0,0720 & 1,000 \\
\hline$P S_{\text {mono }}^{18.100}$ & 47,42 & 0,0780 & 0,9965 & 56,2 & 0,0620 & 1,000 & 68,3 & 0,0718 & 1,000 \\
\hline$P S_{\text {mono }}^{29.100}$ & 47,44 & 0,0673 & 0,9161 & 56,5 & 0,0620 & 1,000 & 68,5 & 0,0715 & 1,000 \\
\hline$P S_{\text {mono }}^{41.200}$ & 47,22 & 0,065 & 0,9049 & 56,3 & 0,0610 & 1,000 & 68,1 & 0,0700 & 1,000 \\
\hline$P S_{p o l i 3}^{18.100}$ & 52,18 & 0,1101 & 0,9878 & 56,1 & 0,0620 & 1,000 & 68,3 & 0,0718 & 1,000 \\
\hline$P S_{\text {polis }}^{18.100}$ & 49,58 & 0,1026 & 0,9831 & 56,1 & 0,0623 & 1,000 & 68,3 & 0,0723 & 1,000 \\
\hline$P S_{p o l i 7}^{18.100}$ & 53,70 & 0,1338 & 0,9602 & 55,6 & 0,0618 & 1,000 & 67,7 & 0,0715 & 1,000 \\
\hline$P S_{p o l i 3}^{107.200}$ & 54,36 & 0,1145 & 0,9966 & 33,3 & 0,0090 & 0,999 & 68,1 & 0,0688 & 1,000 \\
\hline$P S_{p o l i 5}^{107.200}$ & 50,80 & 0,1023 & 0,9980 & 32,0 & 0,0090 & 0,998 & 66,6 & 0,0666 & 1,000 \\
\hline$P S_{\text {poli }}^{107.200}$ & 50,78 & 0,1065 & 0,9920 & 30,4 & 0,00825 & 0,999 & 64,9 & 0,0630 & 1,000 \\
\hline i- $-\mathrm{PP}_{1}$ & 44,46 & 0,1038 & 0,9935 & 33,6 & 0,0277 & 0,899 & 51,9 & 0,0513 & 0,985 \\
\hline $\mathrm{i}-\mathrm{PP}_{2}$ & 42,94 & 0,1039 & 0,9943 & 36,7 & 0,0373 & 0,979 & 54,1 & 0,0600 & 1,000 \\
\hline LLDPE & 39,05 & 0,0725 & 0,9982 & 37,9 & 0,0360 & 0,999 & 47,1 & 0,0417 & 1,000 \\
\hline
\end{tabular}

função da distribuição de massa molar para ambas teorias. Isto não foi observado experimentalmente. Foi mostrado que $-\frac{\partial \gamma}{\partial T}$ aumenta com o aumento da polidispersidade e diminui com o aumento da massa molar (ver Tabela 4). $-\frac{\partial \gamma}{\partial T}$ corresponde a mudança de entropia de formação de superfície (polímero/ar) por unidade de área a volume constante. Os valores obtidos teoricamente para sistemas polidispersos e com massa molar pequena são menores do que os obtidos experimentalmente. Portanto, parece que a teoria está superestimando a mudança de entropia de formação de superfície. Os valores de $-\frac{\partial \gamma}{\partial T}$ obtidos teoricamente (vide Tabela 4) para as poliolefinas são menores do que aqueles obtidos experimentalmente. Isto, provavelmente, é devido ao fato das amostras de poliolefinas serem polidispersas e que a teoria subestima a mudança de entropia de formação de superfície por unidade de área para sistemas polidispersos.

\section{Influência da Massa Molar}

A Figura 4 apresenta a tensão superficial em função da massa molar para poliestireno. Os diferentes símbolos representam os dados experimentais e as curvas as predições teóricas. Pode ser visto da Figura 4 que a tensão superficial de PS aumentou quando a massa molar de poliestireno variou de 3.400 a 41.200 $\mathrm{g} / \mathrm{mol}$. A Figura 4 sugere também que a influência da massa molar na tensão superficial diminui significativamente quando a massa molar excede $41.200 \mathrm{~g} / \mathrm{mol}$ (o valor em que o entrelaçamento ocorre para o $\mathrm{PS}^{[21]}$ ). Comportamento similar foi observado por Kamal et $a l . .^{[22]}$, Ellingson ${ }^{[23]}$ e Arashiro e Demarquette ${ }^{[24]}$ que estudaram a influência da massa molar de PS na tensão interfacial entre PS e PP, PS e PMMA e PS e PE, respectivamente. Os resultados parecem indicar que para massas molares acima de $107.200 \mathrm{~g} / \mathrm{mol}$ a tensão superficial diminui quando a massa molar aumenta. Este fenômeno é provavelmente devido a degradação térmica, uma vez que o tempo de equilíbrio de uma gota pendente de PS com uma massa molar de $200.600 \mathrm{~g} / \mathrm{mol}$ é superior a 6 horas devido a alta viscosidade do polímero. Apesar dos experimentos serem realizados sob atmosfera de argônio, a degradação térmica provavelmente pode ocorrer ${ }^{[13]}$. Esta degradação térmica envolve um aumento da distribuição de massa molar da amostra provocando uma redução da tensão superficial. Neste trabalho, a degradação só foi observada para amostras com massa molar acima de $100.000 \mathrm{~g} / \mathrm{mol}$.

Pode ser visto também na Figura 4 que ambas teorias Patterson e Rastogi e Dee e Sauer prevêem que a tensão superficial aumenta em função da massa molar. A teoria de Dee e Sauer parece superestimar a tensão superficial enquanto que a teoria de Patterson e Rastogi parece subestimá-la. Esta discrepância pode ser corrigida ajustando o parâmetro $m$ e $b$ da teoria ${ }^{[20]}$.

Para ambas teorias $m$ é um parâmetro ajustável que quantifica a parte entálpica da tensão superficial. Logo, as predições teóricas foram ajustadas para PS monodisperso (para massa molar acima da massa 


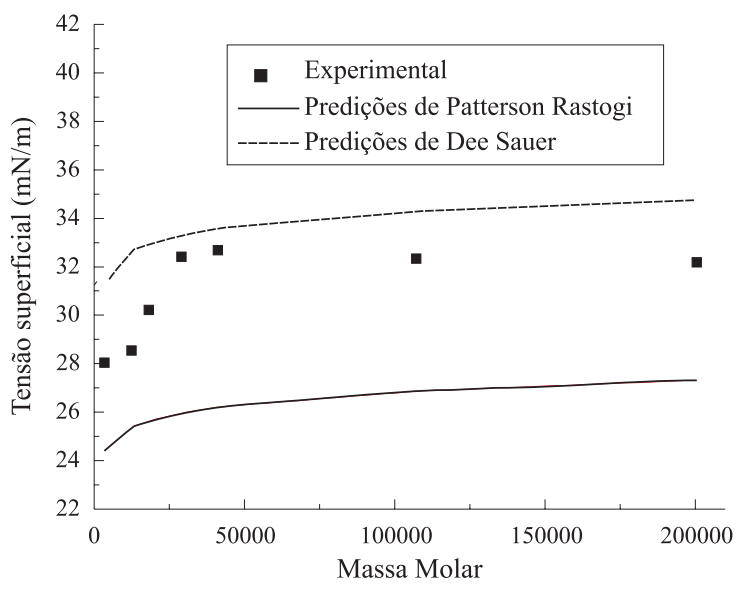

Figura 4. Tensão superficial vs massa molar de PS

molar de entrelaçamento), PP e LLDPE a fim de encontrar os valores de $\mathrm{m}$ de melhor ajuste.

Os valores de m para ambas teorias estão na Tabela 5. Se o excesso de grupos finais não exibisse efeito sobre a tensão superficial, com estes valores de $\mathrm{m}$, as teorias deveriam concordar com os dados experimentais ainda para amostras de baixa massa molar e polidispersa. Este não foi o caso. Por isso, numa segunda etapa, as predições teóricas foram ajustadas aos dados experimentais para encontrar $b$ para cada tipo de polímero e temperatura (b quantifica a contribuição entrópica para a tensão superficial). Isto não foi possível com a teoria de Patterson-Rastogi porque $\mathrm{b}$ pode somente adotar dois valores discretos, 0,89 e 1 . Os valores de b obtidos estão reportados na Tabela 6 . Pode ser visto que $\mathrm{b}$ diminui com o aumento da massa molar para massas molares menores do que a massa molar de entrelaçamento para PS e que b aumenta com $\mathrm{o}$ aumento da polidispersidade. Em outras palavras, a contribuição teórica da entropia para a tensão superficial deveria ser uma função do número de grupos finais que não exibisse um efeito desprezível sobre a tensão superficial. A influência de grupos finais sobre a tensão superficial de polímeros fundidos foi previamente estudada por Koberstein e colaboradores $^{[25]}$ que estudaram a tensão superficial de poli(dimetilsiloxano) com grupos finais modificados de elevada energia de superfície em relação a cadeia principal. Pode ser visto também da Tabela 6 que b aumenta com o aumento da temperatura para todos os polímeros. Dee e Sauer sugeriram que b está relacionado com a espessura da camada interfacial de acordo com a equação (4). Portanto, uma comparação entre os dados experimentais e teóricos de tensão superficial parece indicar que a espessura da camada inter-
Tabela 5. Valores de m

\begin{tabular}{ccc}
\hline Polímero & Patterson-Rastogi & Dee-Sauer \\
\hline PS & 0,27 & 0,51 \\
PP & 0,26 & 0,49 \\
LLDPE & 0,26 & 0,48 \\
\hline
\end{tabular}

Tabela 6. Valores de b

\begin{tabular}{cccc}
\hline Polímero & $\mathbf{4 5 3 ~ K}$ & $\mathbf{4 7 3} \mathbf{~ K}$ & $\mathbf{4 9 3} \mathbf{~}$ \\
\hline$P S_{\text {mono }}^{3.400}$ & 2,4 & 2,4 & 2,5 \\
$P S_{\text {mono }}^{12.400}$ & 2,8 & 2,7 & 2,9 \\
$P S_{\text {mono }}^{18.100}$ & 2,2 & 2,3 & 2,4 \\
$P S_{\text {mono }}^{29.100}$ & 2,0 & 2,0 & 2,0 \\
$P S_{\text {poli3 }}^{18.100}$ & 2,7 & 2,8 & 3,2 \\
$P S_{\text {polis }}^{18.100}$ & 2,9 & 3,3 & 3,4 \\
$P S_{\text {poli7 }}^{18.100}$ & 3,2 & 4,3 & 4,5 \\
$P S_{\text {poli3 }}^{107.200}$ & 2,6 & 2,8 & 3,1 \\
$P S_{\text {poli5 }}^{107.200}$ & 3,0 & 3,3 & 3,4 \\
$P S_{\text {poli }}^{107.200}$ & 3,3 & 3,4 & 3,9 \\
\hline
\end{tabular}

facial é uma função dos números de grupos finais e da temperatura aumentando quando a massa molar diminui e com a distribuição de massa molar.

Contudo, a diferença entre os valores teóricos e experimentais de tensão superficial parece ser de diferente magnitude para alta e baixa massa molar.

Os resultados experimentais de tensão superficial para poliestireno parece seguir uma lei de potências dada por:

$$
\gamma=\gamma_{\infty}-C M_{n}^{-z}
$$

onde $\gamma$ é a tensão superficial, $\gamma_{\infty}, \mathrm{C}$ e z são constantes. $\gamma_{\infty}$ refere-se ao valor limite da tensão superficial para a massa molar infinita, $\mathrm{C}$ reflete a dependência da tensão superficial sobre a massa molar.

Pode ser visto na Tabela 7 que $\mathrm{z}$ foi variado de $1 / 2$ a 1 sem mudança na qualidade do ajuste.

Tabela 7. A influência da massa molar na tensão superficial de PS: parâmetros da equação (8)

\begin{tabular}{cccccc}
\hline $\boldsymbol{z}$ & $\begin{array}{c}\text { Faixa de Massa Molar } \\
\left(\overline{\mathbf{M}}_{\mathbf{n}}: \mathbf{g} / \mathbf{m o l}\right)\end{array}$ & $\begin{array}{c}\text { Temperatura } \\
\left({ }^{\circ} \mathbf{C}\right)\end{array}$ & $\gamma_{\infty}$ & $\boldsymbol{C}_{\boldsymbol{l}}$ & $\boldsymbol{r}^{2}$ \\
\hline \multirow{2}{*}{$1 / 2$} & $12.400-41.200$ & 180 & 37,17 & 137.714 & 0,9847 \\
& & 200 & 36,86 & 164.151 & 0,9706 \\
& & 220 & 34,74 & 155.469 & 0,9820 \\
$2 / 3$ & \multirow{2}{*}{$12.400-41.200$} & 180 & 35,65 & $2,0 \times 10^{9}$ & 0,9793 \\
& & 200 & 35,01 & $2,0 \times 10^{9}$ & 0,9364 \\
& & 220 & 33,00 & $2,0 \times 10^{9}$ & 0,9583 \\
1 & \multirow{2}{*}{$12.400-41.200$} & 180 & 37,17 & 68.857 & 0,9847 \\
& & 200 & 36,86 & 82.075 & 0,9706 \\
& & 220 & 34,74 & 77.734 & 0,9820 \\
\hline
\end{tabular}




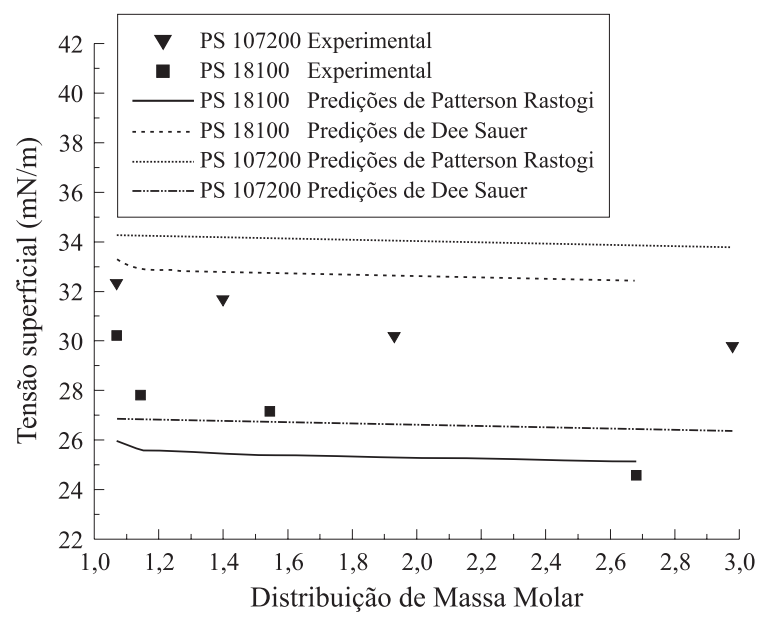

Figura 5. Tensão superficial vs distribuiçao de massa molar de PS

\section{Influência da Distribuição de Massa Molar}

A Figura 5 apresenta a tensão superficial de PS em função da distribuição de massa molar. Os diferentes símbolos representam os dados experimentais e as linhas as predições teóricas.

Pode ser visto na Figura 5 que a tensão superficial de poliestireno diminui em função da distribuição de massa molar de poliestireno quando a massa molar numérica média foi mantida constante. Para uma massa molar numérica média de 18.100 a tensão superficial a uma temperatura de $503 \mathrm{~K}$, diminui em $18,7 \%$ quando a polidispersidade aumenta de 1,08 a 2,68. Para uma massa molar numérica média de 107.200 a tensão superficial a uma temperatura de $503 \mathrm{~K}$, diminui em $16 \%$ quando a polidispersidade aumenta de 1,07 a 2,98 (vide Tabela 4).

Pode ser visto também na Figura 5 que ambas teorias de célula prevêem que a tensão superficial diminui linearmente com o aumento da polidispersidade o que não é observado experimentalmente. Também a magnitude da diminuição da tensão superficial, quando a distribuição de massa molar aumenta, é muito menor teoricamente do que experimentalmente. A diminuição da tensão superficial com o aumento da polidispersidade é devida a um aumento da entropia de formação de superfície quando a distribuição de massa molar aumenta.

\section{Conclusões}

Neste trabalho a influência da temperatura, da massa molar e da distribuição de massa molar na tensão superficial de poliestireno, polipropileno e polietileno linear de baixa densidade foi comparada com as predições teóricas de Patterson e Rastogi e Dee e Sauer. Foi mostrado que ambas teorias prevêem que a tensão superficial de poliestireno, polipropileno e polietileno linear de baixa densidade diminui linearmente com a o aumento da temperatura e aumentam com a massa molar concordando com as tendências observadas nos resultados experimentais. Foi mostrado também que ambas teorias subestimam a mudança de entropia de formação de superfície por unidade de área a volume constante para baixa massa molar, e subestimam a influência da distribuição de massa molar na tensão superficial para poliestireno polidisperso. Além disso, as teorias de Patterson-Rastogi e Dee-Sauer subestimam e superestimam, respectivamente, a tensão superficial. Como m quantifica a contribuição entálpica para a tensão superficial, os dados experimentais para a tensão superficial para PS monodisperso (de uma massa molar maior do que a massa molar de entrelaçamento), PP e LLDPE foram ajustados às teorias para recalcular $\mathrm{m}$ com $\mathrm{b}$ sendo mantido constante. Estes valores de $m$ não resultaram em boa concordância entre os dados experimentais e as predições teóricas para amostras de baixa massa molar e polidispersa. Para uma boa concordância entre os dados experimentais e as predições teóricas, $b$ tinha que ser uma função do número de grupos finais pois $b$ aumenta com a diminuição da massa molar e aumenta com a polidispersidade. Em outras palavras, as teorias deveriam ser modificadas para considerar os efeitos, não-desprezíveis do excesso de grupos finais numa superfície sobre a tensão superficial.

\section{Agradecimentos}

Os autores gostariam de agradecer o Dr. Gregory T. Dee - E. I. DuPont of Nemours and Company, Inc. - por fornecer as propriedades de PVT de poliestireno usadas neste estudo e a FAPESP (97/06071-2, 94/ 3351-6, 97/13505-9) pelo suporte financeiro e pela Bolsa de Estudo de Mestrado de José C. Moreira.

\section{Referência Bibliográfica}

1. Dee, G. T.; Sauer, B. B.; Polymer, 36, 1673 (1995).

2. Dee, G. T.; Sauer, B. B.; Advances in Physics, 47, 161 (1998).

3. Wu, S.: "Polymer Interface and Adhesion", Marcell Dekker, Inc., NY, (1982). 
4. Wu, S; Polymer Eng. Sci., v 27, n 5, p335 (1987).

5. Demarquette, N. R.; da Silva, F. T.; Brandi, S. D.; Gouvêa, D.; Polym. Eng. Sci., (2000).

6. Roe, R. J.; Proc. Nat. Acad. Sci. U.S., 56, 819 (1966).

7. Patterson, D.; Rastogi, A. K.; J. Phys. Chem., v 74, p 1067 (1970).

8. Lennard-Jones, J. E.; Devonshire, A. F.; Proc. R. Soc. London, A, 163, 53 (1937).

9. Flory, P. J.; Orwool, R. A.; Vrij, A.; J. Am. Chem. Soc., v 86, 3507 (1964).

10. Prigogine, I.; Saraga, L.; J. Chim. Phys., 49, 339 (1952).

11. Walsh, D. J.; Dee, G.T.; Halary, J. L.; Ubiche, J. M.; Millequant, M.; Lesec, J.; Monnerie, L.; Macromolecules, 22, 3395 (1989).

12. Moreira, J. C.: “Tensão Superficial de Polímeros Através de Equação de Estado", Dissertação de Mestrado, Universidade de São Paulo, (1999).

13. Moreira, J. C.; Demarquette, N. R.; J. Appl. Polym. Sci., 82, 1907 (2001).

14. Moreira, J. C.; Demarquette, N. R.; SPE ANTEC Tech Papers, 1756 (2000).

15. Arashiro, E. Y.; Demarquette, N. R.; Materials Research, v 2, n 1, p 23 (1999).
16. Anastasiadis, S. H.; Chen, J. K.; Koberstein, J. K.; Siegel, A. F.; Sohn, J. E.; Emerson, J. A.; J. Coll. Inter. Sci., 55, 119 (1987).

17. Demarquette, N. R.; Kamal, M. R.; Polymer Engineering and Science, 34, 24, 1825 (1994).

18. Zoller, P.; Walsh, D.; "Standard Pressure-VolumeTemperature Data for Polymers", Technomic Publishing Company, (1995).

19. Capt, L.; Kamal, M. R.; Intern. Polymer Processing XV, 83, (2000).

20. Demarquette, N. R.; Moreira, J. C.; Shimizu, R. N.; Samara, M. K.; Kamal, M. R.; J. Appl. Polym. Sci., 83, 2201 (2002).

21. Welygan, D. G.; Burns, C. M.; J. Adhes., 14, 169 (1986).

22. Kamal, M. R.; Lai-Fook, R. A.; Demarquette, N. R.; Polym. Eng. Sci., 34, 1834 (1994).

23. Ellingson, P. C.; Strand, D. A.; Cohen, A.; Sammler, R. J.; Carriere, C. J.; Macromolecules, 27, 1643 (1994).

24. Arashiro, E. Y.; Demarquette, N. R.; J. Appl. Polym. Sci., 74, 2423 (1999).

25. Koberstein, J. T.; Duch, D. E.; Hu, W.; Lenk, T. J.; Bhatia, R.; Brwon, H. R.; Lingelser, J. P.; Gallot, Y.; J. Adhes., 66, 229 (1998).

Recebido: 10/08/01 Aprovado: 30/08/02 